\title{
Cervical Cancer Screening Program - A Camp Based Cross Sectional Study Among Rural Women in North Kerala
}

\author{
Usha Karunakaran $^{1,}$ *, Namitha Thekkandathil ${ }^{2}$, Binoo Divakaran ${ }^{1}$, Mini Mol Joseph ${ }^{1}$, \\ Salini Kannankai ${ }^{1}$, Jayasree Anandabhavan Kumaran ${ }^{1}$ \\ ${ }^{1}$ Department of Community Medicine, Academy of Medical Sciences, Pariyaram, Kannur, Kerala \\ ${ }^{2}$ Department of Obstetrics and Gynecology, Academy of Medical Sciences, Pariyaram, Kannur, Kerala
}

\section{Email address:}

ujayadevan@gmail.com (U. Karunakaran),namithaab@gmail.com (N. Thekkandathil), binoovimal@gmail.com (B. Divakaran), minimolramesh@yahoo.co.in (M. M. Joseph), salirajiv@rediffmail.com (S. Kannankai), akjayasree@gmail.com (J. A. Kumaran) ${ }^{*}$ Corresponding author

\section{To cite this article:}

Usha Karunakaran, Namitha Thekkandathil, Binoo Divakaran, Mini Mol Joseph, Salini Kannankai, Jayasree Anandabhavan Kumaran. Cervical Cancer Screening Program - A Camp Based Cross Sectional Study Among Rural Women in North Kerala. Science Journal of Public Health. Vol. 5, No. 3, 2017, pp. 215-223. doi: 10.11648/j.sjph.20170503.19

Received: February 15, 2017; Accepted: March 4, 2017; Published: March 27, 2017

\begin{abstract}
Cervical cancer is ranked as the most frequent cancer in women in India and still remains a leading cause of cancer deaths because of high HPV infection rates and lack of comprehensive cervical Pap smear testing of susceptible women. There has been a regular campaign against cervical cancer for 30 years in India, but impact on the morbidity and mortality has been low, with India ranking fourth worldwide. A key challenge for cancer programs is encouraging women in the 30-55 year age group at highest risk for treatable, precancerous lesions, to seek pap smear screening camps. To increase attendance it is important to work in the community by approaching local women's groups and with their help increasing awareness, knowledge and accessibility of screening among women. In this study the investigators made an effort to increase camp attendance by involving women's groups. We assessed the effect of involvement of women groups in comparison to previous camps, the awareness, attitude and practice of the disease, and its prevention, and the HPV vaccine among the women and finally studied the Pap smear results. A camp based cross sectional study was done in Karindalam village to assess pap smear tests, knowledge, attitude and practice regarding cancer cervix and its screening method. Community level women volunteers were used to mobilise women for the camp. Quantitative data was collected through face to face interview using a pre tested questionnaire and the pap smear test done. Analysis was done with descriptive statistics using epi info. There were 319 women who attended the camp. Out of these $99.7 \%$ were ever married. In our studied population $55 \%$ of the women were married before 20 years of age and $36 \%$ had their first child before 20 years of age. Fifty percent studied up to $10^{\text {th }}$ grade and around $50 \%$ belonged to the lower class. Majority of the women had inadequate knowledge about the disease symptoms $(52.7 \%)$, risk factors $(76 \%)$, preventive measures $(65.5 \%)$, Pap smear method $(91 \%)$ and HPV vaccine $(8.4 \%)$. Reasons for not doing a Pap smear test so far were inadequate knowledge about the disease and test, fear of procedure and result, non availability of the test locally, being non symptomatic, and cultural factors. The Pap smear tests identified $2(0.6 \%)$ women with high intra epithelial lesions, $4(1.2 \%)$, endocervicitis and $3(1 \%)$ with bacterial vaginosisand adequate follow up was offered. The government of India has to respond to the need of the hour and strengthen information, education and communication efforts on cervical cancer along with providing widespread screening facilities.
\end{abstract}

Keywords: Cancer Cervix, Pap Smear Test, HPV Vaccine

\section{Introduction}

Cervical cancer is a malignant neoplasm arising from cells originating in cervix uteri which in the early stages does not cause noticeable symptoms and may be completely asymptomatic. [1] This is a sexually transmitted disease that results from infection with oncogenic types of human papillomavirus (HPV). Oncogenic HPV DNA is found in 
over $95 \%$ of invasive cervical cancers worldwide. [2] It can take as long as 15 to 20 years after the HPV infection for the cancer to develop.

Cervical cancer is ranked as the most frequent cancer in women in India. India has a population of approximately 365.71 million women above 15 years of age, who are at risk of developing cervical cancer. The current estimates indicate approximately 122,844 women are diagnosed with cervical cancer and 67,477 deaths annually in India, accounting to nearly one third of the global cervical cancer deaths. [3] HPV serotypes 16 and 18 account for nearly $76.7 \%$ of cervical cancer in India.[4] Symptoms of cervical cancer may include vaginal bleeding between periods, after sexual intercourse or post-menopausal bleeding, unusual vaginal discharge, pelvic pain radiating to the thighs, pain after sexual intercourse, unexplained weight loss, fatigue, leakage of urine or feces from the vagina and bone fractures. [5, 6]

Other than HPV virus, risk factors including early age at first marriage, longer duration of married life, early and increased parity, low educational status, tobacco consumption, poor genital hygiene, sexual habits and other sexually transmitted diseases, coinfection with HIV, nutritional deficiency, genetic susceptibility and use of hormonal contraceptives, play a significant role in the subsequent development of carcinoma cervix. [7, 8]

Cervical cancer can be prevented by limiting number of sexual partners, using barrier contraceptive methods, avoiding smoking, getting a pap smear and a follow up on abnormalities done, maintaining a healthy diet, a good physical regime and getting the HPV vaccine. [9] Prevention efforts worldwide have focused on screening women at risk of the disease using Pap smears and treating precancerous lesions which can be detected several years before it progresses to invasive cancer. In developed countries, where screening takes place every 3-5 years, the incidence of cervical cancer has been reduced as much as $90 \%$. [10]

Cervical cancer still remains a leading cause of cancer deaths in developing countries because of high HPV infection rates and lack of comprehensive cervical Pap smear testing of susceptible women. To screen successfully, developing countries should have a program that should ensure wide coverage of the target population, guarantee screening, management and adequate follow-up of patients, be provided on-site and be low-cost, with minimum infrastructure requirement that can lead to immediate treatment, if abnormal. A support and funding from the Ministry of Health is also indispensable. [11]

Vaccination against HPV prevents the acquisition of cervical dysplastic lesions among eligible women who have not already acquired the vaccine-specific HPV types. [2] This has not yet acquired much importance in developing countries.

There has been a regular campaign against cervical canal for 30 years in India, but this has had little impact on the morbidity and mortality from the disease, with India ranking fourth worldwide. In India, PAP smear screening camps are available only to a small proportion of women through private health care providers, or in the public sector to women not in the high risk group. Poverty, limited access of the population to information, lack of knowledge of cervical cancer, the absence of sustained prevention programs, lack of healthcare infrastructure required and lack of trained practitioners are the main obstacles to implementation of cervical cancer screening programs. Socio-religious and cultural barriers may also play an important role [11]

If at all implemented, limited or low quality cytological services, diagnostic and treatment services and lack of follow up, may be reasons why screening camps have achieved only limited success. [12]

A key challenge for cancer programs is encouraging women at highest risk for treatable, precancerous lesions, often women in the 30-55 year age group, to seek preventive services. Many women in this age group have completed childbearing and, therefore, are not likely to access family planning or maternal health services. They are also non symptomatic and have no understanding due to lack of knowledge of preventive screening. Special approaches are required to inform them of the need for and availability of screening. The best approaches for increasing awareness among women who are past their reproductive years will vary from place to place. Possible approaches include reaching women through local women's or community groups or linking screening to other midlife health needs, such as contraceptive sterilization (opportunistic screening). For the project to increase the number of women being screened, it is important to work in the community by approaching community leaders and local groups and with their help, increasing awareness, knowledge and accessibility of screening among women. Unlike other types of health screening, such as blood pressure checks, having a cervical screen is something women may feel uncomfortable or embarrassed about. By taking the time to explain the importance of screening, the objectives, and what would be involved, more women feel comfortable attending the screening program. The background work therefore is crucial in engaging people with the issue.

It is also essential to ensure good quality of care at screening sites, treating women with respect and paying attention to their concerns. Program experience from many countries has demonstrated that women will not attend preventive care services, if they believe that they will be treated poorly. [10]

Finally effective cervical cancer prevention ideally requires effective treatment and follow up for which a clear operational link between screening and treatment is necessary. This therefore involves a variety of different interlinked stakeholders, including public health, gynecology, community and women's groups.

In this study, the investigators made an effort to increase camp attendance by involving women's groups. We assessed the effect of this involvement in comparison to previous camps which had no involvement. We also assessed the awareness, attitude and practice of the disease, and its prevention, and the HPV vaccine among the women and finally studied the Pap smear results. 


\section{Materials and Methods}

\subsection{Study Design and Setting}

A cross sectional study with quantitative method of data collection, was conducted in Karindalam, a village in Kasargod, the northern most district of Kerala, a south Indian state. Data collection was conducted from August 1st 2013 to October 31st 2013.

\subsection{Study Population}

The study population was all women aged 20 years and above and who were permanent residents of the village.

\subsection{Method of Data Collection}

In the first stage, the investigators conducted a training program for female volunteers (Anganwadi workers who are members of the Integrated child development scheme (ICDS) and Accredited Social Health Activists (ASHA)) from the same village. They were told in simple terms what cancer cervix was, its signs and symptoms, how it could be prevented, about HPV vaccine, Pap smear and its significance in identifying cancer cervix at a very early stage. They were handed pamphlets to be distributed to women during the home visits. The main purpose of these visits was to motivate women and increase the number of attendees to the cancer screening camps.

In the second stage, cancer screening camps were conducted at three places namely the primary health center at Karindalam and two of their sub centers at Parappa and Choyomkode.. Two days were assigned to each of these centers and thus camps were conducted on six days. A questionnaire which included a section on demography, knowledge of cancer cervix, attitude and practice of attending screening camps and HPV vaccine, motivators for the camp and finally the section on the Pap smear finding was prepared in English after reviewing literature. It was then translated into local language, Malayalam, and back translated into English to check for inconsistencies. A pilot testing was done among 10 women in the reproductive age group and corrections made accordingly. The data collectors were junior residents who were able to communicate in local language. Training was given to the data collectors by the investigators to help them become familiar with data collection instrument. After data collection, brief knowledge about cancer cervix, Pap smear and HPV vaccine was given to the respondents of the camp.

In the third stage, the Pap smear test was done in assigned private rooms within the primary health centers and the sub centers.

In the fourth stage the Pap smears taken back to the Medical college where the investigators worked, were worked upon and the cytological results noted. This was then intimated to the Medical officer of the Primary health center. They identified the women and the results distributed. The investigators then spoke to the concerned women over phone and gave necessary instructions for follow up. Those in need for active treatment were immediately referred to the medical college for further management. Those who needed conservative management were managed at the primary health center itself. Follow up was advised.

\subsection{Operational Definitions}

\subsubsection{Anganwadi Workers}

These are members of the National Integrated child development scheme living in the same village.

\subsubsection{ASHA Workers}

Accredited Social Health Activists of the National rural health mission project living in the same village.

\subsubsection{Permanent Residents}

Those women who lived in that village for more than 6 months continuously.

\subsection{Definitions for Analysis of Data}

For the analysis of data about knowledge, attitude and practice of women related to Pap test, definitions were adopted as described by Gamarra et al as follows: [13]

\subsubsection{Adequate Knowledge}

Women who had heard of cervical cancer and its screening tests and knew the ways/ tests for detecting and preventing cervical cancer.

\subsubsection{Inadequate Knowledge}

Women who had never heard of the cervical cancer and its screening tests, or who had heard of it, but were not aware of screening methods aimed to detect cervical cancer.

\subsubsection{Adequate Attitude}

Women, who considered it as necessary to undergo the screening test for cervical cancer periodically i.e. once in 3 years, which is related to their knowledge on screening policy.

\subsubsection{Inadequate Attitude}

Women who had never heard of the test; women who do not consider it a necessity to undergo the test; women who had no opinion on the matter.

\subsubsection{Adequate Practice}

Women who underwent test within 3 years, prior to data collection.

\subsubsection{Inadequate Practice}

Women who underwent the test more than 3 years prior to data collection or who never had the test. Responses were described in terms of their adequacy.

\subsection{Statistical Analysis}

Descriptive statistics like mean, standard deviation, frequencies and percentages were used. Epi info version 7.0 was used for statistical analysis.

\subsection{Ethical Considerations}

Institutional ethical committee clearance was obtained. 


\subsection{Funding}

Local self Government of Karindalam Panchayat.

\section{Results}

\subsection{Socio Demographic Characteristics}

Three hundred and nineteen women participated in the survey which included a Pap smear screening and also an assessment of knowledge of cancer cervix and attitude and practice of prevention of cancer cervix.

The mean age of the women who participated was 42.46 with a standard deviation of 8.52 years.

Table 1. Age, Religion and caste of the respondents.

\begin{tabular}{lll}
\hline Age & number & percent \\
\hline $20-29$ & 13 & 4 \\
$30-39$ & 121 & 38 \\
$40-49$ & 116 & 36.4 \\
$50-59$ & 58 & 18.1 \\
$60-65$ & 11 & 3.5 \\
Religion & Number & Percent \\
Hindu & 270 & 84.6 \\
Christian & 27 & 8.5 \\
Muslim & 22 & 6.9 \\
Caste & Number & Percent \\
Tribe & 23 & 7.2 \\
Other classes & 296 & 92.8 \\
\hline
\end{tabular}

From table 1 it is seen that $7 \%$ of the women who attended the camps belonged to the 20 to 29 age and above 60 years age category. Eighty four percent of the women were Hindus and tribal women constituted $7.3 \%$ of the attendees. Majority (75\%) were in the 30 to 49 age group.

Table 2. Education, Occupation and Socioeconomic class of the studied population.

\begin{tabular}{lll}
\hline Education & Number & percent \\
\hline Illiterate & 22 & 6.9 \\
Primary & 84 & 26.3 \\
Secondary & 51 & 16 \\
Higher Secondary & 113 & 35.4 \\
+2 & 42 & 13.2 \\
Diploma & 2 & 0.6 \\
Graduate & 5 & 1.6 \\
Occupation & Number & Percent \\
Unemployed & 146 & 45.8 \\
Unskilled & 101 & 31.6 \\
Semiskilled & 43 & 13.5 \\
Skilled & 20 & 6.3 \\
Clerical/ business/farming & 9 & 2.8 \\
Socio Economic Status & Number & Percent \\
Upper middle Class & 84 & 26.4 \\
Middle class & 70 & 22 \\
Lower middle class & 41 & 12.8 \\
Lower class & 124 & 38.8 \\
\hline
\end{tabular}

From table 2 it is seen that $6.9 \%$ of the women are illiterate and $77.7 \%$ have studied up to $10^{\text {th }}$ grade.

Modified B G Prasad socio economic scale 2014 was used to assess the socio economic status of the studied population. [14] Almost 46\% are unemployed/ home makers and $74 \%$ belong to middle and lower class. There were no females in the upper class.

Table 3. Marital status, age of marriage, age of $1^{\text {st }}$ child birth and number of living children of respondents.

\begin{tabular}{|c|c|c|}
\hline Marital Status & Number & Percent \\
\hline Married & 275 & 86.3 \\
\hline Widowed & 34 & 10.6 \\
\hline Separated & 9 & 2.8 \\
\hline Unmarried & 1 & 0.3 \\
\hline Age at marriage & Number & Percent \\
\hline $11-15$ & 15 & 4.7 \\
\hline $16-20$ & 164 & 51.5 \\
\hline $21-25$ & 108 & 33.8 \\
\hline $26-30$ & 24 & 7.5 \\
\hline $31-35$ & 8 & 2.5 \\
\hline Age of $1^{\text {st }}$ child birth & Number & percent \\
\hline No children & 11 & 3.4 \\
\hline $1-15$ & 2 & 0.6 \\
\hline $16-20$ & 112 & 35.2 \\
\hline $21-25$ & 133 & 41.7 \\
\hline $26-30$ & 50 & 15.7 \\
\hline $31-35$ & 8 & 2.5 \\
\hline $36-40$ & 2 & 0.6 \\
\hline $41-45$ & 1 & 0.3 \\
\hline Number of living children & Number & Percent \\
\hline 0 & 11 & 3.4 \\
\hline 1 & 37 & 11.6 \\
\hline 2 & 191 & 60 \\
\hline 3 & 69 & 21.6 \\
\hline 4 & 9 & 2.8 \\
\hline 5 & 2 & 0.6 \\
\hline
\end{tabular}

Table 3 shows that $99.7 \%$ women were ever married and $56.3 \%$ were married before 20 years of age. Nearly $36 \%$ of the women had their first child before 20 years of age. The mean number of children per woman was 2.1 . Twenty five percent had 3 or more children.

Out of the 319 women, 263 were permanently sterilized and therefore not using barrier contraceptive methods, 16 were using barrier methods while 40 were not using contraceptives.

\subsection{Awareness Regarding Cancer Cervix}

Two hundred and eighty two women knew the term cancer cervix.

Table 4. Initial source of knowledge about cancer cervix (No. 282).

\begin{tabular}{lll}
\hline Source of Knowledge & Number & Percent \\
\hline Doctor & 28 & 9.9 \\
ASHA worker & 84 & 29.7 \\
Anganwadi worker & 113 & 40 \\
Family & 9 & 3.3 \\
Friends & 14 & 4.9 \\
Book/magazine & 15 & 5.4 \\
TV & 19 & 6.8 \\
\hline
\end{tabular}

From table 4 it is seen that majority of the women (70\%) got knowledge regarding cancer cervix from women 
volunteers namely Anganwadi and ASHA workers.

Table 5. Knowledge of risk factors of cancer cervix.

\begin{tabular}{lll}
\hline Risk factors mentioned & Number & Percent \\
\hline Infection & 13 & 4.1 \\
Poor hygiene & 14 & 4.4 \\
Repeated coitus & 3 & 0.9 \\
Smoking & 8 & 2.5 \\
Multiparity & 1 & 0.3 \\
Old age & 2 & 0.6 \\
Early marriage & 1 & 0.3 \\
Repeated child birth & 1 & 0.3 \\
Multiple partners & 1 & 0.3 \\
Infection, poor hygiene, early & and repeated & 32 \\
coitus, multiple partners & 243 & 10 \\
Don't know & & 76.3 \\
\hline
\end{tabular}

Only 16 women knew that an organism maybe involved in the causation of disease and out of them only one knew it was a virus. Out of the 319 women, $76.2 \%$ had inadequate knowledge about risk factors of the disease.

Table 6. Knowledge of symptoms of cancer cervix.

\begin{tabular}{lll}
\hline Symptoms & Number & Percent \\
\hline Vaginal discharge & 14 & 4.4 \\
Abnormal vaginal bleeding & 25 & 7.8 \\
Foul smelling vaginal discharge & 6 & 1.9 \\
Intermenstrual bleed & 3 & 0.9 \\
Abdominal pain & 25 & 7.8 \\
Vaginal discharge, bleeding, pain, weight loss, fatigue & 78 & 24.5 \\
Don't know & 168 & 52.7 \\
\hline
\end{tabular}

Nearly 53\% had inadequate knowledge about the symptoms of cancer cervix.

Table 7. Knowledge of measures to prevent cancer cervix.

\begin{tabular}{lll}
\hline Preventive measures & Number & Percent \\
\hline Don't know & 209 & 65.5 \\
Quit tobacco & 5 & 1.6 \\
Vaccine & 27 & 8.5 \\
Regular check up & 18 & 5.7 \\
Good personal and menstrual hygiene & 22 & 6.8 \\
Avoid intercourse at early age & 1 & 0.3 \\
Avoid having multiple sex partners & 2 & 0.6 \\
Regular check up, good personal and menstrual & 35 & 11 \\
hygiene & 35 \\
\hline
\end{tabular}

Sixty six percent had inadequate knowledge about preventive measures of cancer cervix.

Table 8. Knowledge of methods of early detection of cancer cervix.

\begin{tabular}{lll}
\hline Method & No. & Percent \\
\hline Biopsy & 1 & 0.3 \\
See doctor at regular intervals & 86 & 26.9 \\
Scanning & 3 & 0.9 \\
PAP smear & 28 & 8.8 \\
Don't know & 201 & 63.1 \\
\hline
\end{tabular}

Only 28 women $(8.8 \%)$ had heard of the pap smear. Sixty three percent had inadequate knowledge about Pap smear test.

When asked for the necessity of early screening, 203 women $(63.6 \%)$ stated it was for complete treatment and cure.

Regarding vaccine against cancer cervix, only 27 women $(8.4 \%)$ had heard of a vaccine given prophylactically for cancer cervix.

\subsection{Motivation for Camp Attendance}

Table 9. Motivators for camp attendance.

\begin{tabular}{ll}
\hline Motivators & No. (\%) \\
\hline Health Workers & $49(15.4)$ \\
AWW & $53(16.7)$ \\
ASHA Workers & $113(35.4)$ \\
Illness & $44(13.7)$ \\
family members motivated by volunteers & $60(18.8)$ \\
\hline
\end{tabular}

From table 9 it is seen that $52 \%$ of the women were motivated by women volunteers and almost $20 \%$ motivated by family members who were present when the volunteers went to their homes.

\subsection{Attitude and Practice}

Among the 319 women, 28 women had a history of undergone a PAP smear test before. Nineteen women had it done within past 5 years, 6 women had done it 5 years before but within 10 years, while 3 women had it done 20 years ago. Twenty seven women had it done only once before while 1 had it done twice before. This was done as a part of opportunistic screening.

Attitude regarding screening was adequate since all the women who attended the camp wanted the Pap smear test.

Table 10. Reasons cited for not having done a screening test before $(N=$ 291).

\begin{tabular}{ll}
\hline Reason & No. (\%) \\
\hline Non availability of the test & $205(70.5)$ \\
No symptoms & $43(14.8)$ \\
Fear of the result & $30(10.3)$ \\
Fearing pain during the test & $10(3.4)$ \\
Unsupportive Family members & $3(1.0)$ \\
\hline
\end{tabular}

Inadequate knowledge, low motivation and psycho social issues are the one mentioned as reasons for not having done the Pap smear test till date. Even though only $8.4 \%$ of the women had heard of the vaccine, yet $217(68 \%)$ expressed their willingness to take the vaccine.

Two hundred and fifty two women said they would advice their relatives or friends to take it. Out of the 319 women 205 wanted it free of cost from the government. Though knowledge regarding vaccine was inadequate, attitude was good.

\subsection{Pap Smear Results}

Table 11. Pap Smear Results.

\begin{tabular}{ll}
\hline Results & No. (\%) \\
\hline Normal & $239(75 \%)$ \\
Atrophic changes & $31(9.7 \%)$ \\
Erosion cervix & $40(12.6 \%)$ \\
Endocervicitis & $4(1.2 \%)$ \\
Bacterial vaginosis & $3(0.9 \%)$ \\
High grade intraepithelial lesion & $2(0.6 \%)$ \\
\hline
\end{tabular}


Among the 319 women screened $2(0.6 \%)$ had high grade intraepithelial lesion, $3(1 \%)$ had bacterial vaginosis and 4 $(1.2 \%)$ had endocervicitis.

\section{Discussion}

Cervical cancer is an important area of action for any cancer control program, especially so in developing countries because of the burden of disease. Without screening programs cervical cancers are detected at advanced stages, when no curative treatment is possible. This accounts for the poor survival of the cervical cancer patients in the country. Cervical cancer deaths occur at a relatively young age when women are at the prime of their life. [15]

Approximately $80 \%$ of these deaths occur in developing countries. There is a potential for effective prevention via screening. In spite of cervical cancer being preventable, most women in poorer countries are either not aware or do not have access to effective screening programs.

In many middle-income developing countries, cervical screening exists as opportunistic programs and often associated with maternal or child health services, and/or as a component of private health care for affluent women. Opportunistic programs are only partially effective, as coverage does not extend to the majority of women at high risk of the disease. Moreover, they are decentralized and sometimes partially funded.

Cervical cancer screening programs conducted as camps must achieve high compliance to be cost effective and efficient, yet in countries like India, participation is low. Low coverage results in low motivation to conduct such camps in the future and this cycle has a relevant impact on cervical cancer incidence.

As high participation in screening is the primary goal of any organized screening camp, more attention is being paid to strategies to engage and increase number of participants in screening programs. It is seen from studies that several countries in the world are using various strategies like health education, personal invitation by phone calls, by letters, distributing pamphlets, removing screening fee etc., to increase attendance at cervical cancer camps. [16-19]

The investigators have attempted to conduct cervical screening camps earlier. Past experience of the investigators and other studies done in India show low participation in such conducted camps. The major reasons cited by women for not attending cervical cancer screening camps are lack of knowledge, being asymptomatic, fear of the screening test, fear of the result, shyness, hesitation and various sociocultural reasons. [20]

According to WHO, the essential elements for successful cervical cancer screening are to have a decision to initiate cervical screening in the context of a national cancer control program, the political will to support with a funding from the government, an adequate health care infrastructure. In addition, the target population should be well defined, they should be reached out to and educated about the disease and means to identify disease at an early stage, invited to participate in the screening camps, assuring privacy and safety. The other key elements are giving training to health care professionals, defining a referral system for women with abnormal cytology, follow proper patient management guidelines and follow up of patients. [21]

In this study the funding was by the government through the local self government. The local government hospital staff helped the investigators implement the screening program by providing the site at the government hospital and its subcenters and also manpower.

In our study women volunteers namely Anganwadi workers and ASHA workers were selected to mobilize the women from the community. The investigators taught them about the importance of screening for cervical cancer. Later, they went around the village distributing pamphlets and educating the women of the village about cervical cancer and its prevention. In addition, they also invited them to attend the Pap smear camps that were to take place in the ensuing days, promising privacy and safety.

The camps which were conducted on 6 days at three places in a single village had an attendance of 319 women and $70 \%$ were motivated by the women volunteers directly or indirectly. Camps such as these had only achieved an attendance rate of 20 to 30 in the past. From this, it is clear that mobilizing women with the help of community workers is a very good strategy to increase the attendance in screening camps. Camps which have used volunteers to mobilize women have achieved success in cervical screening camps in India. [22]

The mean age of the women was 42.46 years with a standard deviation of 8.52 years. Out of these $99.7 \%$ were ever married. among A survey of 15 to 45 year old rural women in Kerala show that almost $20 \%$ marry by 18 years. [23] In our studied population which include a wider age range (15-65), 55\% of the women were married before 20 years of age and $36 \%$ had their first child before 20 years of age. D. K. Dutta et al (1990) observed that relative risk of acquiring disease was six times more in cases of women who had early parity.[24] Moreover the women did not have any previous opportunity to attend cervical screening camps. Due to these reasons. women belonging to age group $20-30$ and $60-65(7 \%)$ were included in the study. Another reason for inclusion was to ensure attendance in future organized camps. There are studies in India which have included $60-$ 65 year old women in cervical cancer screening camps. [20, 25] The mean number of children this studied population had was 2.1 per woman and $25 \%$ had more than 3 children. Around $50 \%$ studied up to $10^{\text {th }}$ standard and $46 \%$ were house wives. Eighty five percent were Hindus and a few tribal women $(7.3 \%)$ also participated in the study. The Muslim women $(7 \%)$ who participated mentioned that they had to cross some hurdles in their homes to participate in this camp. Studies across the world have shown socio cultural hurdles in cervical cancer screening programs. [26, 27] Socio cultural factors do play a role in community based activities in India also. These women were allowed to attend because the volunteers had promised them that the test would be done by 
lady doctors.

More than $50 \%$ of the women belonged to the lower socio economic status. Generally socio economic status is lower among rural population. People with lower educational status and economic status, living in developing countries have a lesser chance of undergoing pap smear tests compared to urban developed population. [28]

Out of 319, 282 women had heard of the disease and its prevention and 197 (69.7\%) had heard it initially from ASHA and Anganwadi workers during the first stage.

Majority of the women had inadequate knowledge about the disease symptoms $(52.7 \%)$, risk factors $(76 \%)$, preventive measures $(65.5 \%)$, Pap smear method (91\%) and HPV vaccine $(8.4 \%)$. Studies done in India and Kerala have shown inadequate knowledge, attitude and practice among the attendees of cancer cervix screening camps.[29, 30] Forty seven percent knew at least one symptom, 24\% one risk factor and $35 \%$ one preventive measure and this is comparable to a study done in All India Institute of medical sciences. [31] This increase in knowledge was because of the interaction between them and the volunteers. Attitude and practice were also inadequate. Only 28 women $(8.7 \%)$ had done a pap smear before and this was opportunistic. The low coverage could be due to inadequacy in knowledge and no opportunity to attend a screening camp before.

Only $8.4 \%$ had heard about the HPV vaccine and $68 \%$ of them said they would prefer to use it. The rest wanted to know more about the vaccine before deciding. Around $79 \%$ were also willing to advise it to people who have not heard about it. Though awareness about the HPV vaccine was not sufficient, they had adequate attitude about the vaccine. Two hundred and five women (64\%) wanted vaccine free of cost as government supply.

When queried about the reasons for not doing a Pap smear test so far inadequate knowledge about the disease, the test, fear of procedure, non availability of the test in the government hospital and therefore not being prescribed by the doctor, fear of the result, being non symptomatic, shyness and cultural factors were described as the factors. [13, 29]

Finally, maintaining privacy the Pap smear test was done for all the 319 women. The Pap smear tests identified 2 $(0.6 \%)$ women with high intra epithelial lesions, $4(1.2 \%)$ women with endocervicitis and $3(1 \%)$ with bacterial vaginosis. Studies done in India have been able to identify women with high intra epithelial lesions. [32, 33] As soon as the results were known the investigators handed them over to the local government hospital. The concerned women were informed of the result and immediately referred to our Medical college hospital for follow up and treatment. Others who needed treatment (endocervicitis $4(1.2 \%)$ and bacterial vaginosis $3(1 \%))$ were treated at the government hospital itself.

\section{Conclusion}

Though, cervical cancer is the second leading site of cancer among women in Kerala, women in the community are ignorant about the risk factors, symptoms of this particular cancer. Specific knowledge on cervical cancer is thus a critical element in determining whether a woman will undergo Pap test or not. Moreover the primary health care facilities, where cervical screening should be available, are limited, under resourced and over burdened in most developing countries including India. [29]

Thus, in India where a huge section of the population live below poverty line and where awareness among women for cervical cancer control and treatment remain very limited even years after implementation of the National Cancer Control Program in 1975, the recent breakthrough in the global war against cervical cancer does not seem to have much impact. It is overdue that the government responds to the need of the hour and strengthens information, education and communication efforts on cervical cancer along with providing widespread screening facilities. [34]

The HPV vaccine available in India is prophylactic and it works best when given to girls before they begin having sex and run the risk of HPV infection. So, it is clear that the vaccine can in no way be considered a replacement for the standard screening test. [35]

The easy accessibility of the cervix for clinical examination and the existence of clearly defined, easily detectable and easily treatable precursor stages would suggest that standard screening tests would be the gold standard in developing countries. It is important to try and identify factors that might be major obstacles to the effective implementation of such a screening program. The next step would be to develop a program to specifically address those issues. The following are some of the issues that will need to be considered - limited public awareness, limited availability of screening services, inadequate service provider training, inadequate cytological services, inadequate infrastructure, funding, and resources, difficulty in patient follow-up, inadequate follow-up services, inadequate treatment centers, and high cost. Local policy makers, public health professionals, clinicians, cytopathologists, women's groups and health administrators must join hands to develop a program that suits available health resources and medical infrastructure. [36]

Having done that, the following key components like age at primary screening, screening frequency, selection of an appropriate screening test and approach to the management of an abnormal screening result should be considered while developing a national screening program. [36]

\section{Acknowledgement}

This research was supported by the local self government of Karindalam Panchayat. We thank the medical and paramedical staff of Karindalam Primary health center for their support. We also would like to thank the junior staff from Department of Community medicine of our institution for their valuable support in data collection. 


\section{References}

[1] Robbins Basic Pathology, $9^{\text {th }}$ Edition, Vinay Kumar, Abul K. Abbas, Jon Aster.

[2] Ibeanu OA, Molecular pathogenesis of cervical cancer; Cancer Biol Ther. 2011 Feb 1; 11 (3): 295-306.

[3] ICO Information Centre on HPV and cancer (Summary Report 2014-08-22). Human Papillomavirus and Related Diseases in India. 2014.

[4] K. Kaarthigeyan, Cervical cancer in India and HPV vaccination; Indian J Med Paediatr Oncol. 2012 Jan-Mar; 33 (1): 7-12.

[5] Symptoms of cervical cancer, Canadian Cancer society: available at https://www.cancer.ca/en/cancerinformation/cancer-type/cervical/signs-andsymptoms/?region $=$ on.

[6] Signs and symptoms of cervical cancer, American Cancer society: available at https://www.cancer.org/cancer/cervicalcancer/detection-diagnosis-staging/signs-symptoms.html.

[7] Dutta PK, Upadhyay A, Dutta M, et al, A case control study of cancer cervix patients attending Command Hospital, Pune; Indian J Cancer. 1990 Jun; 27 (2): 101-8.

[8] Human Papilloma Virus ICMR: High power Committee to Evaluate Performance of ICMR, 2012-2013. New Delhi, India: ICMR; 2014. Disease Specific Documents for XII plan.

[9] Cherian Varghese, 50 Years of Cancer Control in India; cancer prevention and control in India, Supportive care in Oncology: Historical perspectives and future options; MOHFW India.

[10] Jacqueline Sherris, Cristina Herdman, Christopher Elias, Cervical cancer in the developing world; West J Med. 2001 Oct; 175 (4): 231-233.

[11] Rosa Catarino, Patrick Petignat, Gabriel Dongui, Pierre Vassilakos, Cervical cancer screening in developing countries at a crossroad: Emerging technologies and policy choices, World HYPERLINK

"https://www.ncbi.nlm.nih.gov/pmc/articles/PMC4675913/" J Clin Oncol. 2015 Dec 10; 6 (6): 281-290.

[12] Lazcano-Ponce EC, Moss S, Alonso de Ruíz P, Salmerón Castro J, Hernández AvilaCervical cancer screening in developing countries: why is it ineffective? The case of Mexico. Arch Med Res. 1999 May-Jun; 30 (3): 240-50.

[13] B Das, K Gupta, R Ranjan, M Singh, Knowledge, attitude and practice of cervical cancer screening in women visiting a tertiary care hospital of Delhi; Indian Journal of Cancer, Vol. 51, No. 3, July-September, 2014, pp. 319-323.

[14] Abha Mangal, Varun Kumar et al, Updated BG Prasad socioeconomic classification, 2014: A commentary; Indian Journal of Public Health, 2015 vol 59, Issue 1: 42-44.

[15] Partha Basu, Cervical Cancer Screening in India- Need, Feasibility \& Guidelines for Implementation, available at http://www.bogs.org.in/Review_Article_of_the_month.pdf.

[16] Karla M, Julia C Gage et al, Factors affecting attendance to cervical cancer screening among women in the Paracentral Region of El Salvador: a nested study within the CAPE HPV screening program, BMC Public Health, 2015; 15:
1058.

[17] Laura Camilloni, Eliana Ferroni, Beatriz Jimenez Cendales et al, Methods to increase participation in organised screening programs: a systematic review: BMC Public Health 2013; 13: 464.

[18] Emilia Alfonzo, Agneta Andersson Ellstrom, Szilard Nemes et al Effect of fee on Cervical cancer screening Attendance screen fee, a Swedish Population based Randomised Trial, Plos One: March 17, 2016.

[19] Amelia Acera, Josep Maria Manresa, Diego Rodriguez et al; Increasing cervical cancer screening coverage: A Randomised, Community - based Clinical trial -Plos One; Jan 24, 2017.

[20] Shahina Begum, Naik DD, Sarith Nair et al, Mobilising Women from a low income Community to attend cervical cacer screening camps: Insights from a study in an urban slum of Mumbai, Gynecology and Obstetrics, 2014, Vol 4; Issue 1: 4: 1 .

[21] Report of a WHO Consultation, Cervical cancer screening in developing countries, WHO 2002.

[22] Monali Desai, An Assessment of Community based cancer screening program among Indian women using Anganwadi workers, Obstet Gynecol Ind; Vol 54, No: 5: Sept-Oct 2004. 483-87.

[23] National Family Health Survey 3: 2005-2006.

[24] Dutta P, Upadhyay A, Dutta M et al: A case control study of cancer cervix patients attending command hospital, Pune, Indian J of cancer 1990; 27: 101-8.

[25] GJ Vani, Padmaja, Well Woman Clinic-Screening Program For Cervical Carcinomas. Journal of evolution of medical and dental sciences, Feb 2014, vol 3 issue 8, 1910-14.

[26] Bayrami R, Taghipour A, Ebrahimpour $\mathrm{H}$ et al, Personal and socio cultural barriers to cervical cancer screening in Iran, patient and provider perceptions: A qualitative study; Asian Pac J cancer Prev. 2015; 16 (9): 3729-34.

[27] Michelle S, Williams K, A qualitative assessment of the social cultural factors that influence cervical cancer screening behaviors and the health communication preferences of women in Kumasi, Ghana; J Cancer Educ. 2014 Sept; 29 (3): $555-562$.

[28] Tokin Akinyemiju, Kemi Ogunsina, Swati Sakhuja et al; Lifecourse socioeconomic status and breast and cervical cancer screening: Analysis of the WHO study on Global ageing and adult health (SAGE), BMJ Open. 2016; 6 (11): e012753.

[29] Aswathy S., Mariya Amin Quereshi, Beteena Kurian \& Leelamoni K, Cervical cancer screening: Current knowledge $\&$ practice among women in a rural population of Kerala, India; Indian J Med Res 136, August 2012, pp 205-210.

[30] H N Harsh Kumar, Shubham Tanya, A Study on Knowledge and Screening for Cervical Cancer among Women in Mangalore City, Ann Med Health Sci Res. 2014 Sep-Oct; 4 (5): 751- 756.

[31] Agam B. Bansal, Abhijit B Pakhare, Neelkamal Kapoor et al, Nat Sci Biol Med; Knowledge, attitude, and practices related to cervical cancer among adult women: A hospital- based cross-sectional study, 2015 Jul-Dec; 6 (2): 324-328. 
[32] Sunita A. Bamanikar, Dadaso S. Baravkar, Shirish S. Chandanwale, Prachet Dapkekar, Padmashree; Study of Cervical Pap Smears in a Tertiary Hospital: Indian Medical Gazette - July 2014.

[33] S Yasmin et al; A Cyto-Epidemiological Study on Married Women in Reproductive Age Group (15-49 Years) Regarding Reproductive Tract Infection in a Rural Community of West Bengal: Indian J Public Health 56 (3), 204-209.

[34] M Basu, The relevance of cervical cancer screening and the future of cervical cancer control in India in the light of the approval of the vaccine against cervical cancer; Letter To Editor; Indian Journal of Cancer, Vol. 43, No. 3, JulySeptember, 2006, pp. 139.

[35] K Karthigeyan, Cervical cancer in India and HPV vaccination; Indian J Med Paediatr Oncol, 2012 Jan-Mar; 33 (1): 7-12.

[36] Rajendra A Kerkar, Yogesh V Kulkarni, Screening for cervical cancer: an overview; J Obstet Gynecol India Vol. 56, No. 2: March/April 2006 Pg 115-122. 